\title{
COMMENTARY
}

\section{Breaking old and new paradigms regarding urinary sodium in acute kidney injury diagnosis and management}

\author{
Alexandre Toledo Maciel1,2
}

\begin{abstract}
Urinary sodium ( $\mathrm{NaU}$ ) is one of the oldest parameters used in the evaluation of azotemia and oliguria. Over the past years, however, it has progressively been considered as obsolete and useless, especially in sepsis. It is common sense that NaU frequently does not correlate well with global renal blood flow. If intrarenal microcirculatory changes are more important in acute kidney injury (AKI) than changes in global renal blood flow, we speculate that decreases in NaU may be viewed as a possible marker of microcirculatory impairment in the kidneys. Recent findings by our group (some not yet published) in which sodium retentive capacity is preserved until advanced stages of $\mathrm{AKI}$ and the observation of decreases in NaU preceding increases in creatinine bring us to conclude that the new paradigm of abolishing NaU consideration from daily approaches to managing patients at risk for AKI must be reevaluated.
\end{abstract}

\section{Introduction}

For decades, urinary sodium $(\mathrm{NaU})$ was used to define the presence of structural damage to the kidneys in the setting of oliguria or azotemia [1,2]. The preserved capacity of the tubules to retain sodium was the physiological basis to interpret low levels of $\mathrm{NaU}$ as a functional response to a low renal perfusion state: socalled 'pre-renal' azotemia. The loss of this capacity by the kidneys was considered a marker of 'acute tubular necrosis' (ATN). In the past, levels of $\mathrm{NaU}$ below $20 \mathrm{mEq} / \mathrm{L}$ were considered markers of pre-renal impairment and above $40 \mathrm{mEq} / \mathrm{L}$ as markers of intrinsic renal disease [2].

*Correspondence: alexandre.toledo@intensimed.com

Disciplina de Emergências Clínicas - Instituto Central do Hospital das Clínicas,

Av. Dr. Enéas de Carvalho Aguiar, 255 sala 5023, CEP: 05403-000, Cerqueira César

São Paulo - SP - Brazil

Full list of author information is available at the end of the article
Recently, 'pre-renal' and 'ATN' paradigms have been frequently criticized [3,4]: first, because many cases classified as ATN lack this finding in histopathological studies [5]; and, second, because increasing knowledge of acute kidney injury (AKI) revealed a dissociation between renal hemodynamics and $\mathrm{NaU}$, especially in sepsis [6]. Therefore, these old paradigms gave place to a new paradigm: that $\mathrm{NaU}$ is useless as a tool in AKI management [7]. The aim of this commentary is to question if this new paradigm should be sustained.

\section{The emergence of the new paradigm}

In 2006, a systematic review [8] revealed that $\mathrm{NaU}$ values were widely variable within and between studies with no consistent values to distinguish normal kidney function, pre-renal azotemia and ATN. A contemporaneous experimental study inducing hyperdynamic sepsis revealed progressively lower levels of $\mathrm{NaU}$ [6]. It was hypothesized that the sodium retentive state was due to loss of glomerular filtration pressure. In fact, progressive decreases in $\mathrm{NaU}$ were accompanied by decreases in creatinine clearance. The authors concluded that $\mathrm{NaU}$ was not a reliable marker of renal perfusion (breaking the old 'pre-renal' paradigm). Since then, a new paradigm has emerged: $\mathrm{NaU}$ must not be used as a diagnostic tool in AKI [7].

\section{Reasons why the new paradigm is misleading}

Dissociation between macrohemodynamics and microhemodynamics in sepsis is common. This phenomenon is the possible explanation for apparently paradoxical increases in the sublingual tissue partial pressure of carbon dioxide $\left(\mathrm{P}_{\mathrm{sl}} \mathrm{CO}_{2}\right.$; a marker of microcirculatory stagnation) in parallel with increasing cardiac output during sepsis [9]. Hence, a similar phenomenon could explain the paradox between an increased renal blood flow and low NaU levels. Glomerular perfusion pressure, not total renal blood flow, is the main determinant for $\mathrm{NaU}$ levels. In inflammatory states, low glomerular perfusion pressure may occur in the presence of increased renal blood flow, with activation of sodium-retaining 
mechanisms. Although tubular injury is an early event in AKI [10], most studies still found low fractional excretion of sodium levels in this context $[11,12]$. We may conclude that too much injury is needed to impair the global tubular capacity to retain sodium. Recent unpublished results by our group also suggest that sodium retention is progressively more intense with increases in AKI severity except in very advanced stages (AKI Network stage 3); we hypothesize that extensive tubular injury jeopardized sodium reabsorption. In our findings, such progressive decreases in $\mathrm{NaU}$ began earlier than increases in creatinine, as described in a case report [13].

\section{Reasons why many previous NaU studies are flawed}

Most studies regarding $\mathrm{NaU}$ in AKI have three main limitations. First, $\mathrm{NaU}$ is measured only once instead of sequentially; as previously demonstrated [6,14], NaU responds fast to acute hemodynamic alterations so that relative alterations in it may be more relevant than an isolated $\mathrm{NaU}$ value. It is important to remember that $\mathrm{NaU}$ has a very large physiological range that depends on numerous variables. Of these, the most likely responsible for an abrupt decrease in $\mathrm{NaU}$ value is a decrease in glomerular filtration rate. Second, $\mathrm{NaU}$ is still treated as a categorical variable [15]; the dynamism of $\mathrm{NaU}$ is lost if $\mathrm{NaU}$ is viewed as ' $<20 \mathrm{mEq} / \mathrm{L}$ ' or ' $>40 \mathrm{mEq} / \mathrm{L}$. This view is overly simplistic and unreliable. Third, $\mathrm{NaU}$ is usually assessed only in the presence of oliguria or azotemia. In a recent article [16], we suggested that urinary electrolyte measurement may alert for the presence of AKI development before increases in creatinine or oliguria. In that study, patients who developed AKI in the first 4 days after admittance to the ICU had significantly lower $\mathrm{NaU}$ values at admission.

\section{Microcirculation: a possible bridge between renal blood flow and $\mathrm{NaU}$}

Low NaU values in AKI can be a sign of microcirculatory impairment in the kidneys. We have observed many critically ill patients with very low $\mathrm{NaU}$ levels on the day that renal replacement therapy was initiated. This is not surprising in the context of multiple organ failure, which may be caused by systemic microcirculatory failure. From this perspective, the lower the $\mathrm{NaU}$, the greater the microcirculatory stress. On the other hand, high $\mathrm{NaU}$ values are more difficult to interpret. There is no well established normal range for $\mathrm{NaU}$. In a study including 10 healthy volunteers, the mean $\mathrm{NaU}$ was $104 \pm 48 \mathrm{mEq} /$ $\mathrm{L}$ [17]. We have also found median $\mathrm{NaU}$ values above $100 \mathrm{mEq} / \mathrm{L}$ at ICU admission in patients who did not develop AKI during the study period [16]. However, high $\mathrm{NaU}$ values can be found in patients with AKI receiving diuretics or in advanced AKI stages.

\section{Conclusion}

As for many other monitoring parameters in critical care medicine, the first step in defining $\mathrm{NaU}$ utility in daily practice is to understand properly what it is saying to us and in which contexts. New paradigms regarding this subject should be carefully reevaluated.

\section{Abbreviations}

AKI, acute kidney injury; ATN, acute tubular necrosis; NaU, urinary sodium.

\section{Competing interests}

The author declares that he has no competing interests.

\section{Author details}

${ }^{1}$ Intensive Care Unit, Department of Medical Emergencies, Hospital das Clínicas University of São Paulo, São Paulo, Brazil. ${ }^{2}$ Intensimed Research Group, Hospital São Camilo-Pompéia, São Paulo, Brazil.

Published: 4 February 2013

References

1. Sporn IN, Lancestremere RG, Papper S: Differential diagnosis of oliguria in aged patients. N Engl J Med 1962, 267:130-132.

2. Miller TR, Anderson RJ, Linas SL, Henrich WL, Berns AS, Gabow PA, Schrier RW: Urinary diagnostic indices in acute renal failure: a prospective study. Ann Intern Med 1978, 89:47-50.

3. Bellomo R, Bagshaw S, Langenberg C, Ronco C: Pre-renal azotemia: a flawed paradigm in critically ill septic patients? Contrib Nephrol 2007, 156:1-9.

4. Uchino S: The meaning of transient azotemia. Contrib Nephro/ 2010, 165:337-344.

5. Rosen S, Heyman SN: Difficulties in understanding human "acute tubular necrosis": limited data and flawed animal models. Kidney Int 2001, 60:1220-1224.

6. Langenberg C, Wan L, Bagshaw SM, Egi M, May CN, Bellomo R: Urinary biochemistry in experimental septic acute renal failure. Nephrol Dial Transplant 2006, 21:3389-3397.

7. Prowle J, Bagshaw SM, Bellomo R: Renal blood flow, fractional excretion of sodium and acute kidney injury: time for a new paradigm? Curr Opin Crit Care 2012, 18:585-592.

8. Bagshaw SM, Langenberg C, Bellomo R: Urinary biochemistry and microscopy in septic acute renal failure: a systematic review. Am J Kidney Dis 2006, 48:695-705.

9. Maciel AT, Creteur J, Vincent JL: Tissue capnometry: does the answer lie under the tongue? Intensive Care Med 2004, 30:2157-2165.

10. Gonzalez F, Vincent F: Biomarkers for acute kidney injury in critically ill patients. Minerva Anestesio/ 2012, 78:1394-1403.

11. Nejat M, Pickering JW, Devarajan P, Bonventre JV, Edelstein CL, Walker RJ, Endre $\mathrm{ZH}$ : Some biomarkers of acute kidney injury are increased in pre-renal acute injury. Kidney Int 2012, 81:1254-1262.

12. Bagshaw SM, Haase M, Haase-Fielitz A, Bennett M, Devarajan P, Bellomo R: A prospective evaluation of urine microscopy in septic and non-septic acute kidney injury. Nephrol Dial Transplant 2012, 27:582-588.

13. Maciel AT, Park M: Early diagnosis of acute kidney injury in a critically ill patient using a combination of blood and urinary physicochemical parameters. Clinics(Sao Paulo) 2012, 67:525-526.

14. Caironi P, Langer T, Taccone P, Bruzzone P, De Chiara S, Vagginelli F, Caspani L, Marenghi C, Gattinoni L: Kidney instant monitoring (K.IN.G): a new analyzer to monitor kidney function. Minerva Anestesiol 2010, 76:316-324.

15. Bagshaw SM, Bennett M, Devarajan P, Bellomo R: Urine biochemistry in septic and non-septic acute kidney injury: a prospective observational study. J Crit Care 2012, pii:S0883-9441(12)00334-6.

16. Maciel AT, Park M, Macedo E: Urinary electrolyte monitoring in critically ill patients: a preliminary, observational study. Rev BrasTer Intensiva 2012, 24:236-246.

17. Masevicius FD, Tuhay G, Pein MC, Ventrice E, Dubin A: Alterations in urinary strong ion difference in critically ill patients with metabolic acidosis: a prospective observational study. Crit Care Resusc 2010, 12:248-254.

doi:10.1186/cc11926

Cite this article as: Maciel AT: Breaking old and new paradigms regarding urinary sodium in acute kidney injury diagnosis and management. Critical Care 2013, 17:115. 